\title{
LA-8444-MS
}

Informal Report

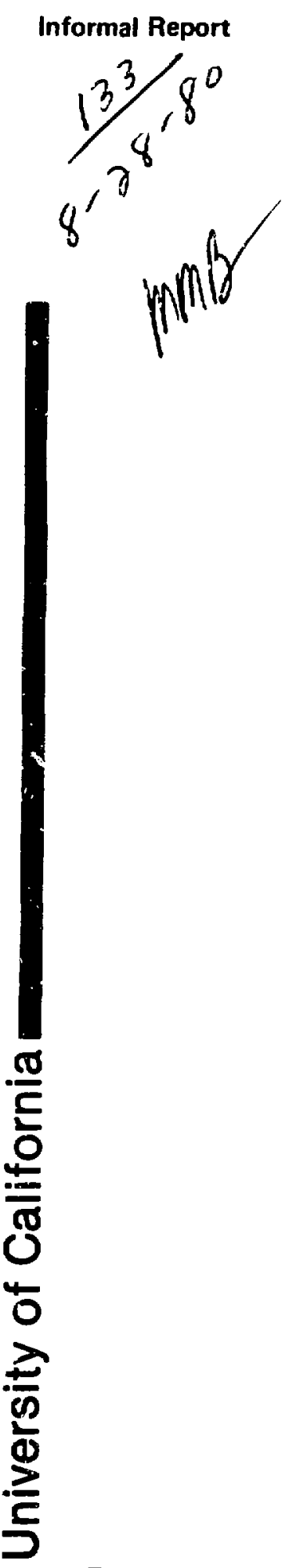

\section{A Monochromatic Neutral Kaon Beam}

竞

$\frac{2}{\mathrm{c}}$

$\stackrel{\frac{2}{D}}{5}$
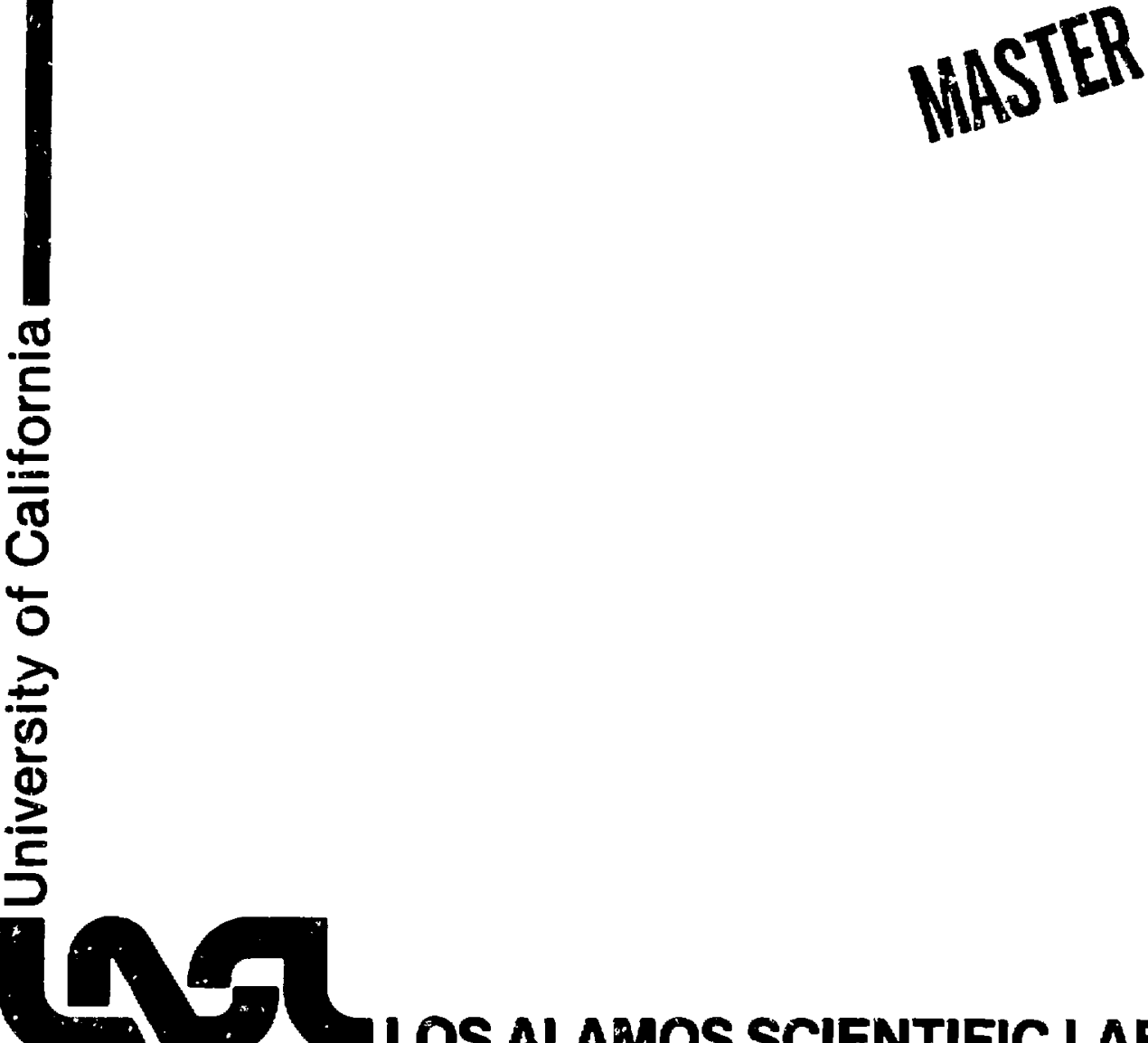
LA-8444-MS

Informal Report

UC-34c

Issued: July 1980

\title{
A Monochromatic Neutral Kaon Beam
}

\author{
Cyrus M. Hoffman
}
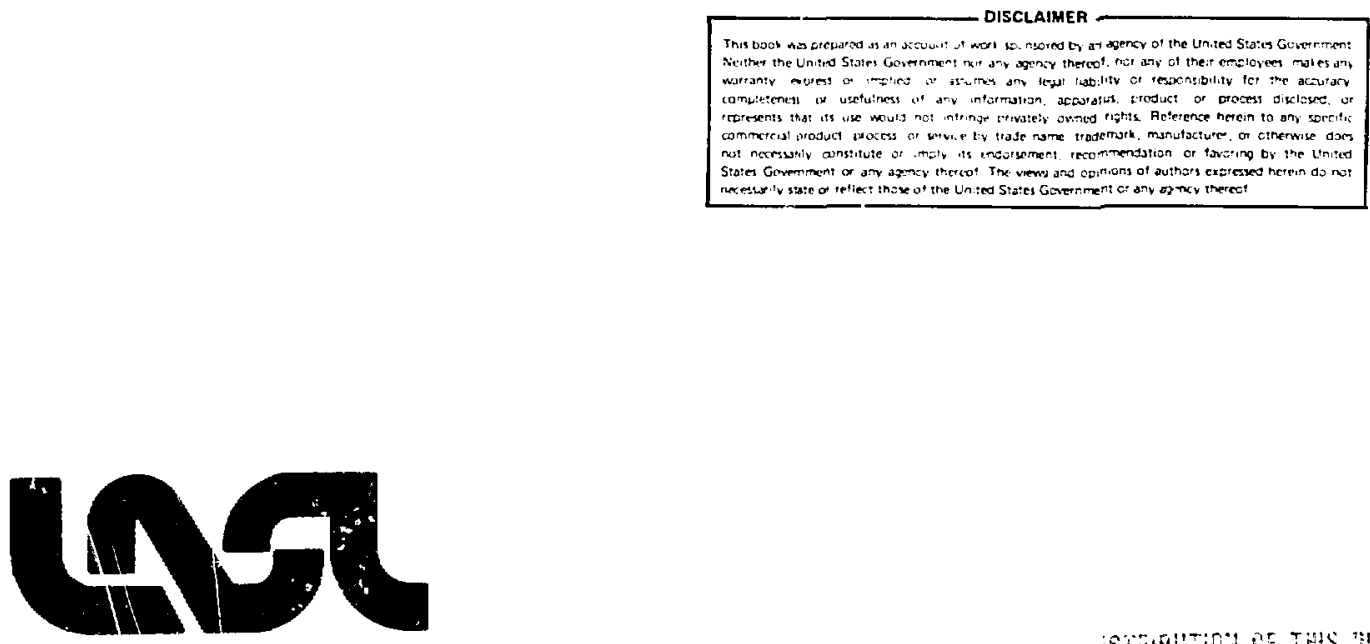
A MONOCHROMATIC NEUTRAL KAON BEAM

by

Cyrus M. Hoffman

\begin{abstract}
A design for a monochromatic, neutral kaon $\left(K^{0}\right)$ beam, which could be constructed at a "Kaon Factory," is described. Possible uses of such a beam are discussed.
\end{abstract}

\title{
I. INTRODUCTION
}

Neutral kaon bearsis have been plagued by large neutron backgrounds and large energy spreads. The large neutron fluxes increase the singles rates in detectors and so increase background rates. This can prevent the placing of detectors or other material in the beam which can make experiments difficult, if not impossible. The large $K^{0}$ energy spread implies an experimental design which 1 flexible enough to accommodate the range of energies and precludes the use of the reconstructed $K^{0}$ energy as a constraint. We describe a scheme whereby a monochromatic $K^{0}$ beam 1 might be produced at a "kaon factory" 2 which would have only modest neutron contamination. Some potential uses of such a beam are discussed.

\section{EXISTING $K^{0}$ BEAMS}

We are now in a period where no low-to-medium energy $K^{0}$ beams exist in the world. There are no $K^{0}$ beams at CERN, ${ }^{3}$ KEK, 4 or SLAC, 5 so that, with the closing of the ZGS, the only $K^{0}$ beams are at Serpukhov, Fermilab and at the AGS. The neutral beams at Serpukhov and Fermilab are high-energy beams, generally in excess of $50 \mathrm{GeV}$. There are two neutral beams at the AGS. 6 The $A_{3}$ beam produces $210^{6} \mathrm{KL} / \mathrm{pulse}$ with $1<\mathrm{p}<11 \mathrm{GeV} / \mathrm{c}$ and 212 neutrons $/ \mathrm{K}^{0} \mathrm{~L}$. The $B_{5}$ beam is a short beam (2.6-m long) which yields $22 \times 10^{5} \mathrm{KL} / \mathrm{pulse}$ with momenta from 1 to $215 \mathrm{GeV} / \mathrm{C}$ and 15 neutrons $/ K^{0} L$. Other $K^{0}$ beams used to exist at the CERN-PS, the AGS and the ZGS with generally similar parameters.

\section{GENERAL CONCEPT}

All existing $K^{0}$ beams are produced via proton collisions with some heavy nucleus: this accounts for both the large neutron contamination and the large energy spread. The construction of a very high intensity ( $100 \mu \mathrm{A})$, 
20-GeV proton accelerator (a "kaon factory") would make possible tertiary production of monochromatic $\mathrm{K}^{0}$ beams with small neutron cortamination. The basic idea is as follows: the high-intensity proton beam strikes a target producing (among other things) $K^{-1} s$. A short, high-flux separated $K^{-}$beam delivers the $\mathrm{K}^{-}$to a liquid hydrogen target where $\overline{\mathrm{K}}^{\circ}$ 's are produced via the reaction $K^{-} p \rightarrow \bar{K}^{0} n$. Since the $K^{-}$are momentum-analyzed in the $K^{-}$beam section, and the $\bar{K}^{0}$ 's are produced in a two-body reaction, the $\bar{K}^{0}$ 's have a well-defined energy. Neutron contamination should be low since a beam with enriched strangeness is used to produce the neutral kaons.

\section{DESIGN}

In this section we present a candidate beam design and discuss the beam properties which might result. In order to simplify the probiem, we assume that the $\mathrm{K}^{-}$beam section has characteristics somewhere between those of LESB $I$ and LESB II at the AGS: 6 this takes some account of the lower proton energy (20 GeV rather than $28 \mathrm{GeV})$ and is certainly achievable. The parameters of the $\mathrm{K}^{-}$beam are then:

$\begin{array}{ll}\text { Momentum } & 1050 \mathrm{MeV} / \mathrm{c} \\ \mathrm{K}^{-} / 10^{12} \text { protons } & 2 \times 10^{5} \\ \text { Production angle } & 7^{0} \\ \Delta \mathrm{p} / \mathrm{p} & \pm 2 \% \\ \text { Beam spot } & 1 / 2^{\prime \prime} \times 1 / 2^{\prime \prime} \\ \mathrm{K}^{-} / \pi^{-} \text {(After } & 1 / 9 .\end{array}$

The momentum of $1050 \mathrm{MeV} / \mathrm{c}$ is chosen to take advantage of a local maximum in the $\mathrm{K}^{-} \mathrm{p} \rightarrow \bar{K}^{0} \mathrm{n}$ cross section caused by the production of the $\Lambda^{*}$ (1830). The total cross section is $8.3 \mathrm{mb}$ and $\mathrm{d} \sigma / \mathrm{d} \Omega * 10^{\circ}=13.3 \pm 2 \mathrm{mb} / \mathrm{sr} .7$ We will assume a proton current of ? $\times 10^{14}$ protons $/ \mathrm{s}(35 \mu \mathrm{A})$ incident on the production target. This will produce a $\mathrm{K}^{-}$beam with $4 \times 10^{8} \mathrm{~K} / \mathrm{s}$ and $4 \times 10^{9} \pi^{-/} \mathrm{s}$. The number of $\bar{K}^{0} \cdot s$ produced at $0^{\circ}$ by a $1 \mathrm{M}$ liquid hydrogen target is then

$$
\begin{aligned}
\# \overline{\mathrm{K}}^{0} / \mathrm{S} & =\left(\# \mathrm{~K}^{-} / \mathrm{s}\right) \times\left(\rho L \mathrm{LH}_{2}\right) \times\left(\text { Length } L \mathrm{LH}_{2}\right) \times\left(\left.\frac{\mathrm{d} \sigma}{\mathrm{d} \Omega}\right|_{\theta=0}\right) \times\left(\frac{\mathrm{d} \Omega^{*}}{\mathrm{~d} \Omega}\right) \times(\Delta \Omega) \times\left(N_{A V}\right) \\
& =\left(4 \times 10^{8}\right) \times(0.07) \times(100) \times\left(13.3 \times 10^{-27}\right) \times(3.5) \times(\Delta \Omega) \times\left(6 \times 10^{23}\right) \\
& =\left(7.8 \times 10^{7}\right) \times \Delta \Omega(\text { ster }) .
\end{aligned}
$$

where $\Omega^{*}$ refers to the center-of-mass and $\Omega$ to the laboratory. Neutron production will be dominated by $\pi^{-} p \rightarrow \pi^{0} n$ : for neutrons produced at $0^{\circ}$ in the lab, the interesting region is at $\theta_{C M}^{*}=180^{\circ}$. The $\pi^{-} p$ charge exchange cross 
section at $180^{\circ}$ is $0.5 \pm 0.1 \mathrm{mb} / \mathrm{ster}^{8}$ (in the center of mass) or $\sim 3 \mathrm{mb} / \mathrm{ster}$ in the laboratory: the neutron momentum is $1354 \mathrm{MeV} / \mathrm{c}$. The neutron flux is then

$$
\begin{aligned}
\# n / s & =\left(\# \pi^{-} / \mathrm{s}\right) \times\left(\rho \mathrm{LH}_{2}\right) \times\left(\text { Length } L_{2}\right) \times\left(\left.\frac{\mathrm{d} \sigma}{\mathrm{d} \Omega}\right|_{180^{\circ}}\right) \times(\Delta \Omega) \times N_{A V} \\
& =\left(4 \times 10^{9}\right) \times(0.07) \times(100) \times\left(3 \times 10^{-27}\right) \times(\Delta \Omega) \times\left(6 \times 10^{23}\right) \\
& =\left(5 \times 10^{7}\right) \times \Delta \Omega(\text { ster }) .
\end{aligned}
$$

While there will certainly be additional channels contributing to the neutron flux, it is clear that the neutron to $\bar{K}^{0}$ ratio will be $0(1)$, roughly a factor of 10 better than existing beams.

In order to obtain a useful $\bar{K}^{0}$ flux, the neutral beam solid angle must be large; presumably between $10^{-3}$ and $10^{-2}$ steradians. Since the flux incident on the hydrogen target is comparable to the flux used to produce the $B_{5} K^{0}$ beam at the AGS $\left(25 \times 10^{9} / \mathrm{s}\right)$ and the incident energy is significantly lower ( 2 ) GeV vs $28 \mathrm{GeV}$ ), adequate shielding can probably be achieved in less than $2.6 \mathrm{M}$; let's say $2 M$ will be required. We then want a beam spot of from $40 \mathrm{~cm}^{2}$ to $400 \mathrm{~cm}^{2}$, not unreasonable.

The neutral beam will consist of a sweeping magnet to divert the incident $\mathrm{K}^{-} / \pi^{-}$beam away and to sweep out any charged particles produced in the hydrogen target, 5 to 10 radiation lengths of material to convert photons, another sweeping magnet, and collimators to define the beam. The choice of radiator thickness and collimator arrangenent will be flexible since they are not located in high radiation areas.

\section{KO ENERGY SPREAD}

Assuming $\bar{K}^{0}$ production at $0^{\circ}$ from $K^{-} \mathrm{p} \rightarrow \bar{K}^{0} \mathrm{n}$ at $1050 \mathrm{MeV} / \mathrm{c}, \frac{{ }^{\partial} \mathrm{E}_{K^{0}}}{\partial \theta_{\mathrm{Lab}}}=1.5$ MeV/deg. Thus the $\bar{K}^{0}$ energy spread will be dominated by the $\Delta p / p$ in the incident $K$ beam, assumed to be several percent. There will be some low energy $\bar{K}^{0}$ 's from more complicated reactions such as $K^{-} p \rightarrow \bar{K}^{0} p \pi^{0}$ but the cross sections for these reactions are small.9

\section{USES OF BEAM}

The obvious uses of a neutral kaon beam are CP-violation studies. Of special interest are precision measurements of the magnitudes and phases of noo and $n_{+-}$, and the magnitude of the $K_{L}-K_{S}$ mass difference, precision studies of charge asymmetries in $K^{0} \rightarrow \pi^{ \pm} e^{\mp} v$ and $\pi^{ \pm} \mu^{\mp} v$, and searches for $T$ T-violation in $\mathrm{K}^{0} \rightarrow \pi \mu \nu$. Gauge theories of $C P$ violation have been formulated, "O but the source of the $C P$ violation and the strength of $C P$ yiolation ("superweak" or "milliweak") have yet to be settled. Some models ${ }^{10}$ predict deviations from "superweak" predictions which are onty slightly smaller than current experimental resolution. Thus improving the determination of the CP violation parameters is very important. In these measurements and in other experiments, knowledge of the $K^{0}$ energy $r$. ovides an added constraint and would help suppress backgrounds. 
Experimental studies of $K^{0} \rightarrow \pi e \nu$ and $K^{0} \rightarrow \pi \mu \nu$ have been plagued by a well-known two-solution ambiguity. 11 The two solutions correspond to the $v$ going forward or backward in the center of mass. Knowledge of the $K^{0}$ energy resolves the ambiguity. This will be invaluable in studies of this decay mode to study the form factors of the $K-\pi$ vertex, the CP-violating charge asymmetries and the $\Delta S=\Delta Q$ rule. Studies of rare decay modes such as $K_{L} \rightarrow \mu^{+} \mu^{-}$(and measuring the muon polarization) and $K_{L} \rightarrow \mu^{ \pm} e^{\mp 12}$ may require higher $K^{0}$ fluxes.

A monochromatic $\overline{\mathrm{K}}^{0}$ beam also allows $\overline{\mathrm{K}}^{0}-\mathrm{N}$ scattering experiments. These can be useful in determining the $\overline{K N}$ phase shifts.

\section{CAVEATS}

The calculations performed for this study are quite crude. No Monte Carlo studies have been performed; no second-order processes have been considered. Accordingly, all results should be taken in this spirit: they indicate the orders of magnitudes to be expected but certainly may be incorrect by factors of two.

\section{ACKNOWLEDGMENTS}

I benefited from useful conversations with R. E. Mischke, V. D. Sandberg and H. A. Thiessen.

\section{REFERENCES}

1. Throughout this paper the symbol " $K^{0 "}$ will be used to denote either $K^{0}$ or $\overline{\mathrm{K}}^{0}$.

2. Proceedings of the Kaon Factory Workshop, TRIUMF Report TRI-79-1, Vancouver, B. C., Canada, (1979).

3. "Experiments at CERN in 1979," CERN Report, 1979.

4. T. Sato in "Workshop on the AGS Fixed Target Research Program," BNL 50947 (1979).

5. C. G. Wohl et al., LBL Report-91, 1978.

6. G. M. Bunce, BNL 50874 (1978).

7. R. Armenteros et al., Nucl. Phys. BS, 233 (1968).

8. C. B. Chiu $\in t$ al., Phys. Rev. 156, 1415 (1967).

9. Processes such as $K^{-} p \rightarrow \bar{K}^{0} n \pi^{0}$ and $K^{-} p \rightarrow \bar{K}^{0} p \pi^{-}$are roughly an order of magnitude less probable than $K^{-} \mathrm{p} \rightarrow \bar{K}^{0} n$ and do not peak at $\theta_{\bar{K}^{0}}=0^{\circ}$. 
10. See e.g., R. N. Mohapatra in Proceedings of the XIX International Conference on High Energy Physics, Tokyo, 1978 and $L$. Wolfenstein in Proceedings of Neutrino 79, Bergen, 1979.

11. See e.g., V. Bisi, et a1., Phys. Letters 36B, 533 (1971).

12. P. Herczeg in Proceedings of the Kaon Factory Workshop, TRIUMF Report, TRI-79-1, pg. 20 (1979). 